\section{A Uniform Concept for Error Estimation in Gamma-Ray Spectrometry}

\section{Péter Zagyvai, Lajos György Nagy, and József Solymosi}

Technical University of Budapest

Department of Physical Chemistry

Hungary

\section{Introduction}

The estimated errors of peak areas are primarily important results for the complete analytical process (e.g., activation analysis or environmental radiocontamination assay). Many excellent program routines were offered in the literature for determining peak areas; however, no unambiguous solution has been given so far for assigning accurate error estimation methods.

As an introduction to our "uniform concept" some basic principles are to be stated:

- The error estimation would establish an accurate confidence limit around the calculated results: this region would include the "true value." Thus the error is a measure of accuracy rather than that of precision.

- The above condition is met only if the estimated error combines all statistical (random) and systematic (bias) errors.

- The methods for peak area determination and error estimation, respectively, would be closely interdependent.

- The actual formulation is strongly related to the given detection system.

\section{Theory}

Of course it is necessary that a uniform method be used for calculating peak areas of singlets and multiplets as well. The "apportioning" method proved satisfactory for resolving overlaps:

$$
I_{\mathrm{i}}=\frac{a_{\mathrm{i}} \cdot s_{\mathrm{j}}}{\sum_{\mathrm{j}=1}^{K} a_{\mathrm{j}} \cdot s_{\mathrm{j}}} \cdot I_{\mathrm{ST}}
$$

where

$I_{\mathrm{ST}}$ is the total count rate of the multiplet determined by summing and baseline subtraction,

$K \quad$ is the number of overlapping peaks

$I_{\mathrm{i}}$ is the count rate of the i'th peak,

$a$ is the net peak height, and

$s \quad$ is the peak width.

Peak heights are computed from least squares fit with fixed and previously calibrated widths. The error of the $i$ 'th count rate is given by eq (2):

$$
\left(\Delta I_{\mathrm{i}}\right)^{2}=\frac{\left(\Delta a_{\mathrm{i}}\right)^{2}}{a_{\mathrm{i}}^{2}}+\frac{\sum_{\mathrm{j}=\mathrm{1}}^{K}\left(\Delta a_{\mathrm{j}}\right)^{2}}{\left(\sum_{j=1}^{K} a_{\mathrm{j}}\right)^{2}}+\frac{\left(\Delta I_{\mathrm{ST}}\right)^{2}}{I_{\mathrm{ST}}^{2}} \cdot I_{\mathrm{i}}^{2}
$$

The first two terms represent the "bias" error, the third one contains the "random" error. Peak height errors $\Delta a$ are generated in the least squares fit.

The count rate $I$ for singlets is the maximum of the summed and baseline-subtracted net counts $\left(I_{S}\right)$ and the integral of the fitted peak shape function $\left(I_{\mathrm{F}}\right)$. If we consider this step as a "one component apportioning," the error of the count rate is given by eq (3):

$$
(\Delta I)^{2}=\frac{(\Delta a)^{2}}{a^{2}}+\frac{(\Delta a)^{2}}{a^{2}}+\frac{\left(\Delta I_{\mathrm{S}}\right)^{2}}{I_{\mathrm{S}}^{2}} \cdot I^{2}
$$

The resemblance of eqs (2) and (3) is quite clear.

If the radioactivities attributed to the identified isotopes are calculated not individually but in a common least squares procedure (called interference correction), the estimated peak area errors must be applied as weight factors in the least squares solution.

\section{Results}

Numerous reference materials were analyzed to confirm the reliability of both the peak area computation and error estimation methods (such as Bowen's kale, NBS and IAEA reference materials). In testing the experimental standard deviations, it was revealed that some analytical results were biased. However, most results were still reliable because the known true values in most cases did fall into the calculated confidence intervals. 


\section{Summary}

We elaborated a uniform formulation for calculating estimated count rate errors in gamma spectrometry. Experimental results confirmed that our method gave accurate confidence limits combining both random and bias errors.

\section{Accuracy in $C P A A$ for $C, N$ and $O$ and in ERDA and NRA for $H$}

\author{
T. Nozaki \\ Department of Hygienic Sciences \\ Kitasato University \\ Kitasato, Sagamihara, \\ Kanagawa 228, Japan
}

\section{Introduction}

Trace amounts of $\mathrm{H}, \mathrm{C}, \mathrm{N}$ and $\mathrm{O}$ can be determined by only a few methods, often with rather poor accuracies. Charged particle activation analysis (CPAA) is highly reliable for $\mathrm{C}, \mathrm{N}$ and $\mathrm{O}$, and is used for calibration of other methods. Elastic recoil detection analysis (ERDA) and nuclear reaction analysis (NRA) have recently been utilized for $\mathrm{H}$ and $D$ near the surface. Accuracies in these analyses are discussed on the basis of our experimental data.

\section{Accuracy in CPAA}

\subsection{Advantage and Error in CPAA}

The advantage of CPAA for $\mathrm{C}, \mathrm{N}$ and $\mathrm{O}$ consists of inherent high sensitivity, freedom from various contaminations, and reliability in the use of comparators. The following reactions are used for activation: ${ }^{12} \mathrm{C}\left({ }^{3} \mathrm{He}, \alpha\right){ }^{11} \mathrm{C}$ or ${ }^{12} \mathrm{C}(\mathrm{d}, \mathrm{n}){ }^{13} \mathrm{~N} ;{ }^{14} \mathrm{~N}(\mathrm{p}, \alpha){ }^{11} \mathrm{C}$; and ${ }^{16} \mathrm{O}\left({ }^{3} \mathrm{He}, \mathrm{p}\right){ }^{18} \mathrm{~F}$. Uncertainty in the measurement of bombarding particle beam current frequently becomes a major cause of error in CPAA. Also, some bombardment damage of the sample often results in noticeable overestimation of $\mathrm{C}$ and $\mathrm{O}$.

\subsection{Precision for $\mathbf{O}$ and $\mathrm{C}$}

We analyzed $O$ in a silicon wafer repeatedly nine times from 1982 to 1985 by the nondestructive measurement of the ${ }^{18} \mathrm{~F}$ annihilation radiation with a $\mathrm{Ge}(\mathrm{Li})$ detector [1]. The results gave the mean value of $10.04 \mathrm{ppm}$ wt with $\sigma=0.18 \mathrm{ppm}$.

We examined chemical separation of ${ }^{11} \mathrm{C}$ in $\mathrm{Si}$, using ${ }^{11} \mathrm{C}$-containing $\mathrm{Si}$ prepared by proton bombardment of B-doped Si [1]. The following separation method was selected: alkali-dissolution of the pulverized sample; $\mathrm{KMnO}_{4}$ oxidation of the ${ }^{11} \mathrm{C}$ under microwave heating to $700{ }^{\circ} \mathrm{C}$; and generation of ${ }^{11} \mathrm{CO}_{2}$ and its conversion into $\mathrm{Li}_{2}{ }^{11} \mathrm{CO}_{3}$. The measurement of ${ }^{11} \mathrm{C}$ activity before and after the separation and of carrier recovery indicated that $9.0 \pm 2.0 \%$ of the ${ }^{11} \mathrm{C}$ apparently disappeared. Our results obtained by this separation has thus been corrected for this $9 \%$.

For the separation of ${ }^{13} \mathrm{~N}$, dry fusion into ${ }^{13} \mathrm{~N}_{2}$ and wet distillation as ${ }^{13} \mathrm{NH}_{3}$ are used [1]. Carbon in 27 plates of GaAs made from a single rod were analyzed by the dry method in different machine times of two cyclotrons. The mean results were $24.6 \mathrm{ppb}$ wt with $\sigma=2.6 \mathrm{ppb}$. For the last five plates taken from adjacent parts of the rod, the results were $23.25 \pm 0.84 \mathrm{ppb}$. We separate ${ }^{18} \mathrm{~F}$ by precipitation as $\mathrm{KB}^{18} \mathrm{~F}_{4}$ [2]. This method has shown satisfactory precision.

\subsection{Calibration of IR Spectrophotometry}

Our calibration curve for IR spectrophotometry of $\mathrm{C}$ in $\mathrm{Si}$ is shown in figure 1 [1]. About 70 carefully prepared samples were submitted to roundrobin IR measurement in 24 organizations. About $1 / 3$ of them were then analyzed by CPAA; some of them were also analyzed by SIMS. The differences between the results of CPAA and SIMS are probably caused by the presence of background in SIMS. Satisfactory calibration curves were obtained also for $\mathrm{O}$ and $\mathrm{N}$ in $\mathrm{Si}[3,4]$. Efforts are now being made to obtain a reliable calibration curve for $\mathrm{C}$ in $\mathrm{GaAs}$.

\section{Accuracy for ERDA and NRA for $\mathrm{H}$}

Figure 2 shows ERDA spectra of a $\mathrm{H}$ - and Dcontaining amorphous silicon film on a $\mathrm{Si}$ wafer [5]. The detection efficiency is seen to depend highly on the detection angle. No suitable substances are found as comparators for $\mathrm{H}$ and $\mathrm{D}$, and their quantities are usually obtained in comparison with an internal standard element by the use of the theoretical relationships and experimental parameters. The $\mathrm{H}$ content of amorphous silicon films obtained by ERDA and vacuum fusions are given in 